\title{
Radical Polymerization of $p$-Substituted Styrenes with Benzyl Phenyl Selenide as Photoiniferter
}

\author{
Tae Seok Kwon, Hiromitsu OchiaI, Shuji Kondo, ${ }^{\dagger}$ Koji TaKagI, \\ Hideo Kunisada, and Yasuo $\mathrm{YUKI}^{\dagger \dagger}$ \\ Department of Materials Science and Engineering, Nagoya Institute of Technology, \\ Gokiso-cho, Showa-ku, Nagoya 466-8555, Japan
}

(Received August 3, 1998)

\begin{abstract}
Benzyl phenyl selenides were used as photoiniferters for polymerizations of $p$-bromostyrene, $p$-chloromethylstyrene, $p$-methylstyrene, and $p$-vinylbenzyl acetate. In the limited range of conversion ( $\leqq 50 \%)$, polymer yields and number-average molecular weights increased with polymerization time. Polymers from $p$-methoxybenzyl $p$-trimethylsilylphenyl selenide contained the benzyl and arylseleno groups at $\alpha$ - and $\omega$-chain ends, respectively. Photoirradiation of polystyrene containing a phenylseleno group at the $\omega$-chain end as polymeric photoiniferter afforded effectively block copolymers of styrene and $p$-substituted styrenes.

KEY WORDS Radical Polymerization / Photoiniferter / Benzyl Phenyl Selenide / $p$-Bromostyrene / p-Chloromethylstyrene / p-Methylstyrene / p-Vinylbenzyl Acetate / Polymeric Photoiniferter / Block Copolymer
\end{abstract}

Organoselenium compounds play important roles in biological systems and modern organic synthesis. ${ }^{1-3} \mathrm{We}$ recently studied the synthesis and polymerization of selenium-containing monomers. ${ }^{4}$ We examined the synthesis of polystyrene containing the seleno group at both chain ends using diphenyl diselenide and the preparation of end functional polymers by elimination reaction, a representative reaction of organoselenium chemistry. ${ }^{5,6}$ More recently, we reported the synthesis of the end functional polystyrene and poly(methyl methacrylate) by radical polymerization in the presence of benzyl phenyl selenide (BPSE-1) as photoiniferter. ${ }^{7}$ The resulting polystyrene (1PS) had benzyl and phenylseleno groups at $\alpha$-and $\omega$-chain ends, respectively (eq 1). Photoirradiation of this polymeric photoiniferter in the presence of methyl methacrylate (MMA) afforded a block copolymer (1PS-b-PMMA) of styrene (St) and MMA (eq 2). The resulting block copolymer had low block efficiency, possibly to the low-end functionality ( $D F=0.5-0.6$ ) for MMA. ${ }^{8}$ BPSE-1 should thus function as a photoiniferter in the radical polymerization of $p$-substituted styrenes and most resulting polymers have seleno group at terminal chain ends.

Poly(bromostyrene) is an intermediate to prepare functional polymers containing various groups such as thiol, sulfide, carboxylic acid, and others. ${ }^{9-12}$ Poly-

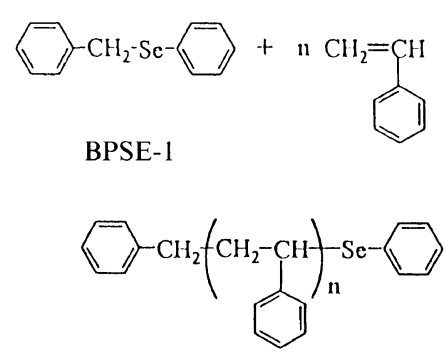

h $v$

IPS

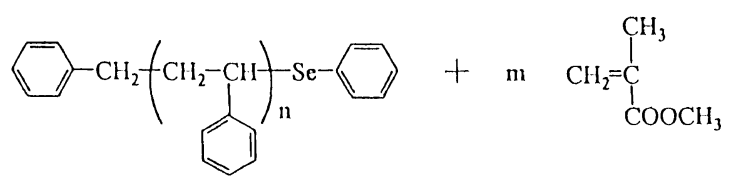

IPS

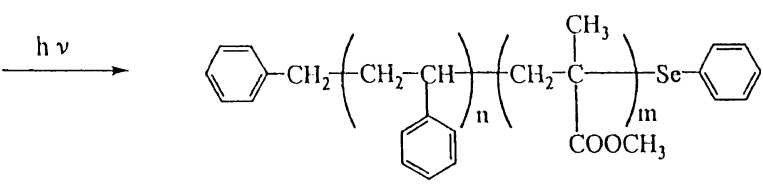

1PS- $b$-PMMA

(chloromethylstyrene) is an important precursor in the synthesis of arborescent polymers. ${ }^{13}$ Poly(vinyl benzyl acetate) is of interest as a precursor of poly(vinyl benzyl alcohol). ${ }^{14,15}$

This paper describes the radical polymerization of p-bromostyrene (BS), $p$-chloromethylstyrene (CMS), $p$ methylstyrene (MS), and $p$-vinylbenzyl acetate (VBAc) in the presence of BPSE-1 and the synthesis of AB-type block copolymers of end functional polystyrene (1PS) and poly( $p$-substituted styrene). The synthesis of ABC-type triblock copolymer from AB-type block copolymer as a polymeric photoiniferter with BS is also investigated.
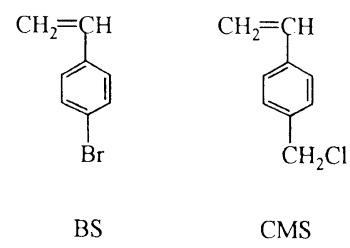<smiles>C=Cc1ccc(C)cc1</smiles><smiles>C=Cc1ccc(CC(=O)OC(C)=O)cc1</smiles>

CMS MS

VBAc

\footnotetext{
$\dagger$ Deceased August 5, 1997.

${ }^{\dagger \dagger}$ To whom all correspondence should be addressed (Fax: +81-52-735-5294).
} 


\section{EXPERIMENTAL}

\section{Materials}

St, CMS, and MS were purified by the ordinary method and distilled in a stream of nitrogen just before use. BS was prepared from $p$-bromobenzene by acetylation, reduction, and dehydration. ${ }^{16}$ VBAc was prepared from CMS and potassium acetate. ${ }^{17}$ BPSE- $1 \quad\left(\lambda_{\max }=280\right.$, $\varepsilon=14000$ in ethanol) and $p$-methoxybenzyl $p$-trimethylsilylphenyl selenide (BPSE-2) $\left(\lambda_{\max }=280, \varepsilon=20000\right.$ in ethanol) were prepared as in previous papers. ${ }^{7}$ Solvents were purified by distillation after appropriate drying. Other reagents were obtained commercially and used without further purification.

\section{Photopolymerization of p-Substituted Styrene with BPSE-I}

The required amounts of $p$-substituted styrene, BPSE1 , and benzene were charged into a Pyrex tube of $15 \mathrm{~mm}$ diameter. The tube was degassed under vacuum by conventional freeze and thaw techniques and sealed under vacuum. All polymerizations were carried out by irradiation with a Riko $100 \mathrm{~W}$ high pressure mercury lamp at an $8 \mathrm{~cm}$ distance at room temperature. After a given time, the tube was opened and the reaction mixture was poured into methanol for MS, BS, and VBAc, or hexane for CMS. The resulting polymers (PMS-1, PBS-1, PVBAc-1, and PCMS-1) were purified by reprecipitation followed by drying in vacuum.

\section{Chain Extension Polymerization of Poly(p-bromostyrene)}

Chain extension polymerization was examined using poly ( $p$-bromostyrene) $\left(0.6 \mathrm{~g}, \bar{M}_{n}=4500, \bar{M}_{w} / \bar{M}_{n}=2.45\right)$ as polymeric photoiniferter, BS $(9 \mathrm{~mL})$ and benzene $(6 \mathrm{~mL})$ were divided equally into six polymerization tubes. The solutions were irradiated with a high-pressure mercury lamp at room temperature. After polymerization, the reaction mixture was poured into methanol. The resulting polymer was dried in vacuum.

\section{Synthesis of Polystyrene (IPS) as a Polymeric Photo- iniferter}

A solution of BPSE-1 $(0.24 \mathrm{~g})$, styrene $(3 \mathrm{~mL})$ in benzene $(2 \mathrm{~mL})$ was immersed in a Pyrex tube. The tube was degassed under vacuum by conventional freeze and thaw techniques, and sealed under vacuum. The solution was irradiated with the $100 \mathrm{~W}$ high pressure mercury lamp for $10 \mathrm{~h}$. The tube was opened and contents were poured into methanol. 1PS was reprecipitated from methylene chloride to methanol. (Yield, $0.48 \mathrm{~g}, 17.7 \%$ ).

\section{Synthesis of $A-B$ Block Copolymers}

A solution of 1PS $(0.06 \mathrm{~g}), p$-substitute styrene $(3 \mathrm{~mL})$, and benzene $(2 \mathrm{~mL})$ in Pyrex tube was irradiated with a high-pressure mercury lamp at room temperature for $20 \mathrm{~h}$ or $30 \mathrm{~h}$. The polymer mixture was poured into methanol. The fraction of the polymer was confirmed by GPC and ${ }^{1} \mathrm{H}$ NMR spectra. The composition of block copolymers were examined by GPC and ${ }^{1} \mathrm{H}$ NMR spectra for block copolymers of 1PS with CMS, MS, and VBAc and block copolymers of 1PS with BS, it was evaluated from elementary analysis of bromine.
Table I. Photopolymerization ${ }^{a}$ of MS, BS, VBAc, and CMS in the presence of BPSE-1

\begin{tabular}{|c|c|c|c|c|c|}
\hline Monomer ${ }^{b}$ & $\begin{array}{c}\text { [Monomer] } \\
\text { [BPSE-1] }\end{array}$ & $\frac{\text { Time }}{h}$ & $\frac{\text { Yield }^{\mathrm{c}}}{\%}$ & $\bar{M}_{n}^{\mathrm{d}}$ & $\bar{M}_{w} / \bar{M}_{n}$ \\
\hline MS & 300 & 5 & 4.6 & 1600 & 2.43 \\
\hline MS & 300 & 10 & 8.6 & 2400 & 2.32 \\
\hline MS & 300 & 15 & 11.0 & 2900 & 2.44 \\
\hline MS & 300 & 20 & 13.4 & 3300 & 2.56 \\
\hline BS & 500 & 3 & 9.1 & 6000 & 2.05 \\
\hline BS & 500 & 6 & 17.3 & 7100 & 2.11 \\
\hline BS & 500 & 8 & 21.2 & 7600 & 2.18 \\
\hline BS & 500 & 10 & 22.8 & 8200 & 2.20 \\
\hline BS & 500 & 12 & 29.2 & 9600 & 2.28 \\
\hline VBAc & 500 & 6 & 5.3 & 5400 & 2.70 \\
\hline VBAc & 500 & 10 & 9.3 & 7000 & 2.75 \\
\hline VBAc & 500 & 18 & 19.6 & 12000 & 2.63 \\
\hline VBAc & 500 & 22 & 22.9 & 13400 & 2.57 \\
\hline CMS & 100 & 5 & 5.3 & 2200 & 2.50 \\
\hline CMS & 100 & 10 & 13.1 & 3400 & 2.27 \\
\hline CMS & 100 & 15 & 18.8 & 4300 & 2.20 \\
\hline CMS & 100 & 20 & 26.4 & 5300 & 2.13 \\
\hline CMS & 100 & 25 & 36.4 & 5700 & 2.10 \\
\hline
\end{tabular}

${ }^{a} 100 \mathrm{~W}$ high pressure $\mathrm{Hg}$ lamp. ${ }^{\mathrm{b}}[\mathrm{Monomer}]_{0}=4.0 \mathrm{~mol} \mathrm{~L}^{-1}$ in benzene. ${ }^{\mathrm{c}}$ Precipitated with methanol for MS, BS, and VBA polymers and $n$-hexane for CMS polymers. ${ }^{\mathrm{d}}$ Estimated by GPC.

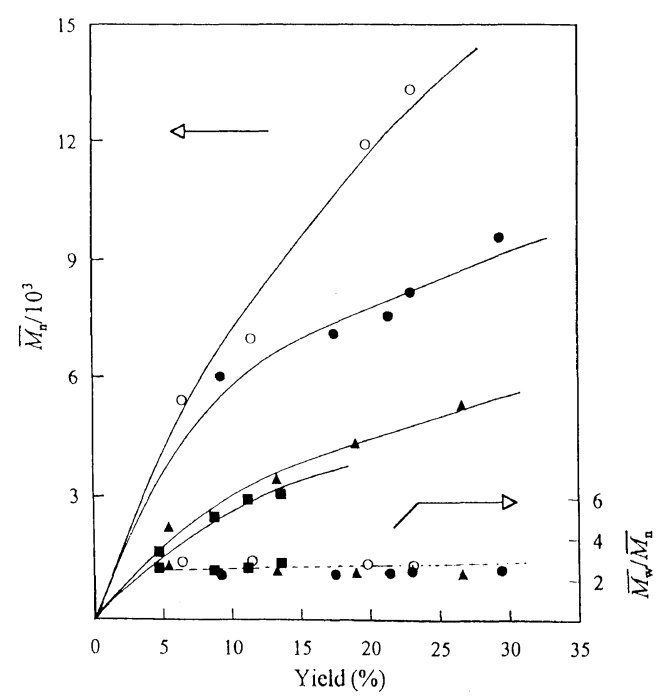

Figure 1. Yield $-\bar{M}_{n}$ and yield $-\bar{M}_{w} / \bar{M}_{n}$ relations for the photopolymerization of MS $(\boldsymbol{\square})$, BS $(\boldsymbol{O})$, VBAc $(\bigcirc)$, and CMS $(\boldsymbol{\Delta})$ with BPSE-1 as a photoiniferter. [ $p$-Substituted styrene] $=4.0 \mathrm{~mol} \mathrm{~L}^{-1}$ in benzene.

\section{Synthesis of $A-B-C$ Block Copolymer}

A solution of BS $(2 \mathrm{~mL})$, benzene $(3 \mathrm{~mL})$ and block copolymer of 1 PS with MS $(0.075 \mathrm{~g})$ in a Pyrex tube was irradiated with the high-pressure mercury lamp at room temperature for $15 \mathrm{~h}$. The polymer mixture was poured into methanol. The yield was $0.82 \mathrm{~g}$. The composition of triblock copolymer was examined by GPC and ${ }^{1} \mathrm{H}$ NMR spectra.

\section{Measurements}

IR spectra were measured by a Nicolet Impact-400D. NMR spectra were recorded by a Varian XL-200 spectrometer with $\mathrm{CDCl}_{3}$ as solvent using tetramethysilane 
Table II. Photopolymerization ${ }^{\mathrm{a}}$ of $p$-substituted styrenes with BPSE- $2^{\mathrm{b}}$ in benzene solution at room temperature

\begin{tabular}{|c|c|c|c|c|c|c|c|c|c|}
\hline \multirow{2}{*}{ Polymer } & \multirow{2}{*}{$\frac{[\text { Monomer }]}{\text { mol L }^{-1}}$} & \multirow{2}{*}{$\frac{\text { Time }}{\mathrm{h}}$} & \multirow{2}{*}{$\frac{\text { Yield }^{\mathrm{c}}}{\%}$} & \multirow{2}{*}{$\bar{M}_{n} / 10^{3}$} & \multirow{2}{*}{$\bar{M}_{w} / \bar{M}_{n}$} & \multirow{2}{*}{$\mathrm{b} / \mathrm{a}^{\mathrm{d}}$} & \multicolumn{2}{|c|}{ Degree of polymerization } & \multirow{2}{*}{$D F^{f}$} \\
\hline & & & & & & & by GPC & by ${ }^{1} \mathrm{H} N \mathrm{NM}^{\mathrm{e}}$ & \\
\hline PBS-2 & 4.0 & 5 & 21.7 & 7.31 & 2.16 & 3.17 & 38.0 & 34.1 & 1.10 \\
\hline PVBAc-2 & 4.0 & 10 & 18.0 & 8.93 & 2.04 & 2.87 & 48.8 & 55.7 & 0.88 \\
\hline PCMS-2 & 4.0 & 15 & 23.6 & 6.57 & 2.35 & 2.92 & 40.7 & 41.5 & 0.98 \\
\hline
\end{tabular}

${ }^{a}$ Irradiation of $100 \mathrm{~W}$ high pressure mercury lamp. ${ }^{b}[$ Monomer $] /[\mathrm{BPSE}-2]=300 .^{\mathrm{c}}$ Precipitation with methanol. ${ }^{\mathrm{d}} \mathrm{b} / \mathrm{a}=$ the relative integral of trimethylsilyl signal at $0.25 \mathrm{ppm}$ (b) to methoxy signal at $3.72 \mathrm{ppm}$ (a) in Figure $2 .{ }^{\mathrm{e}} 400 \mathrm{MHz}$ in $\mathrm{CDCl}_{3}$. ${ }^{\mathrm{f}} \mathrm{The}^{\mathrm{T}}$ degree of functionality (DF) was determined by GPC and ${ }^{1} \mathrm{H}$ NMR.
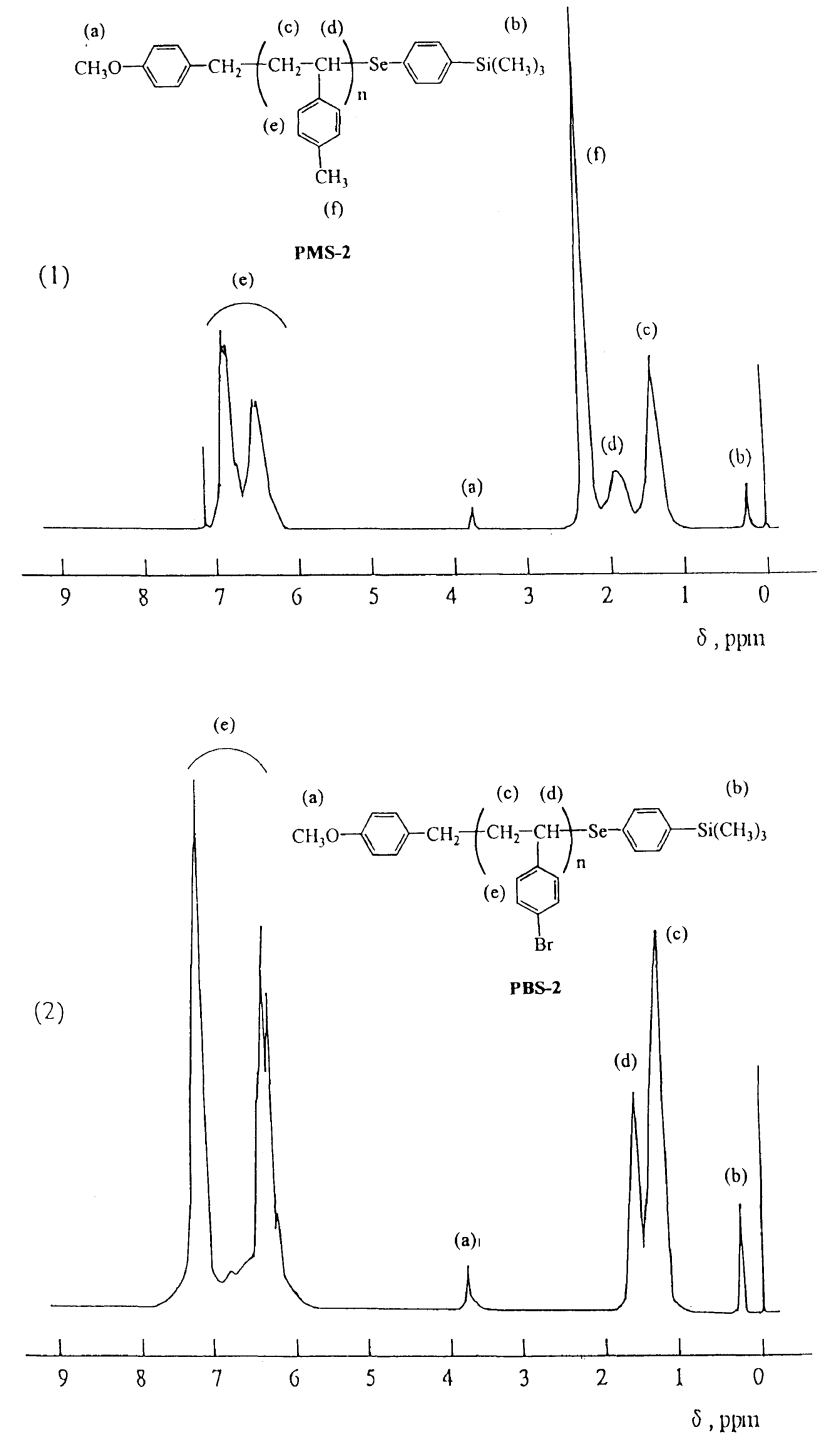

as the internal standard. UV spectra were measured by a JASCO Ubest-35 spectrometer. GPC was carried out on a TOSOH HLC-803D with G2000, G3000, and GMH TSK gel-columns and differential refractometric detector in tetrahydrofuran. Molecular weights were determined based on polystyrene standards.

\section{RESULTS AND DISCUSSION}

\section{Photopolymerization of p-Substituted Styrene}

Polymerizations of BS, CMS, MS, and VBAc were carried out with BPSE-1 as photoiniferter by irradiation
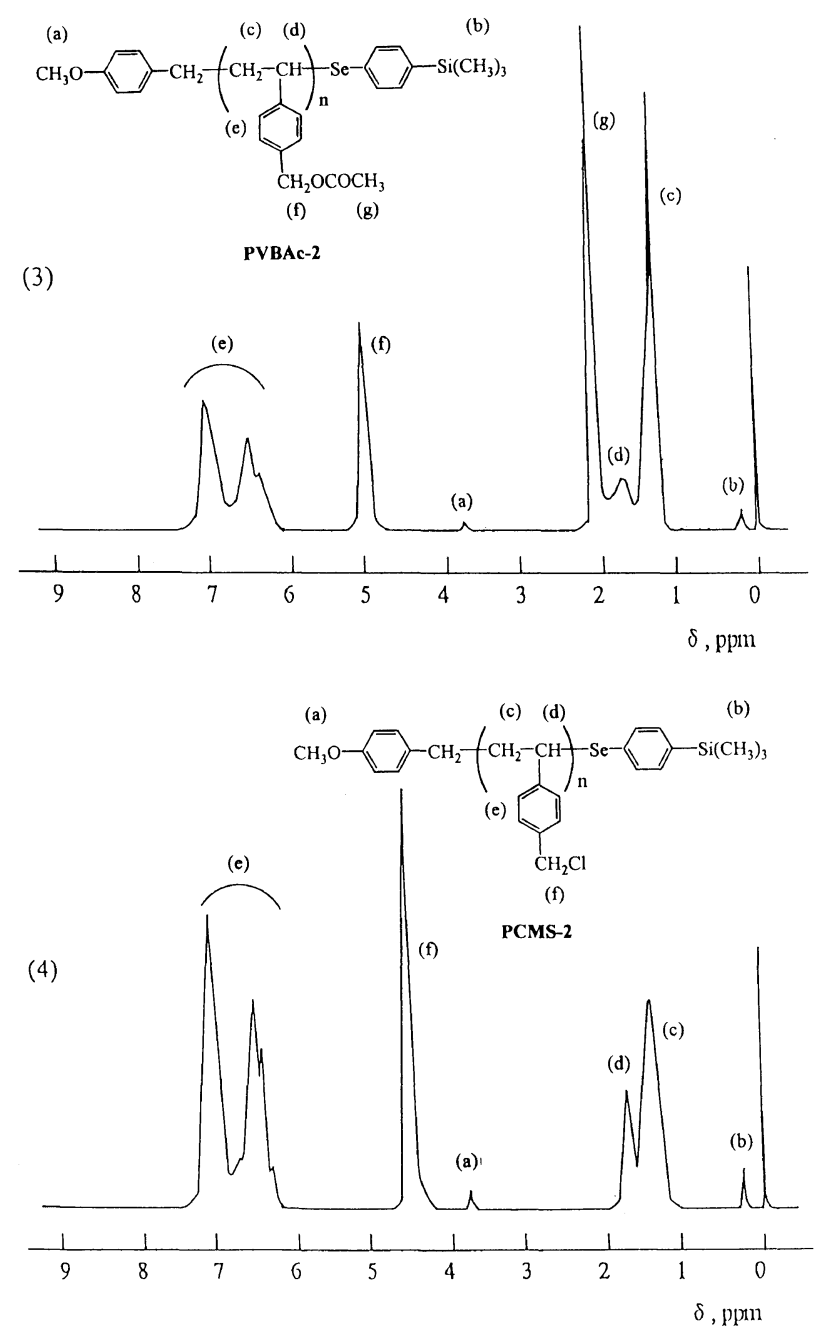

Figure 2. ${ }^{1} \mathrm{H}$ NMR spectra $\left(400 \mathrm{MHz}\right.$, in $\left.\mathrm{CDCl}_{3}\right)$ of (1) PMS-2 $\left(\bar{M}_{n}=6260, \bar{M}_{w} / \bar{M}_{n}=2.15\right),(2) \mathrm{PBS}-2\left(\bar{M}_{n}=7310, \bar{M}_{w} / \bar{M}_{n}=2.16\right),(3)$ PVBAc-2 $\left(\bar{M}_{n}=8930, \bar{M}_{w} / \bar{M}_{n}=2.04\right)$, and (4) PCMS-2 $\left(\bar{M}_{n}=6570\right.$, $\left.\bar{M}_{w} / \bar{M}_{n}=2.35\right)$.

of a high pressure mercury lamp at room temperature. The results are shown in Table I. $\bar{M}_{n}$ was increased with polymerization time in all cases. Figure 1 shows the relations of yield and $\bar{M}_{n} \cdot \bar{M}_{n}$ increased with increasing polymer yield in all cases. Therefore, control of polymerization by BPSE-1 as a photoiniferter is proved not only for St and $\mathrm{MMA}^{7}$ but also $p$-substituted styrenes.

BPSE-2 was used to investigate the end structure of the resulting poly $\left(p\right.$-substituted styrene) because ${ }^{1} \mathrm{H}$ NMR peaks based on methoxy and trimethylsilyl groups did not overlap with those of poly ( $p$-substituted styrene). 


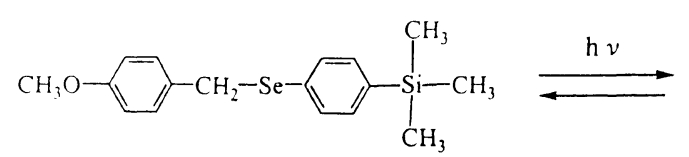

BPSE-2

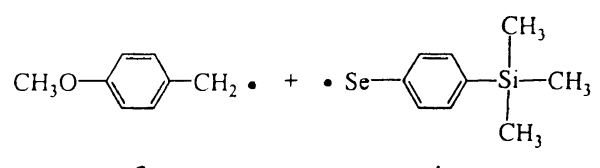

3

4
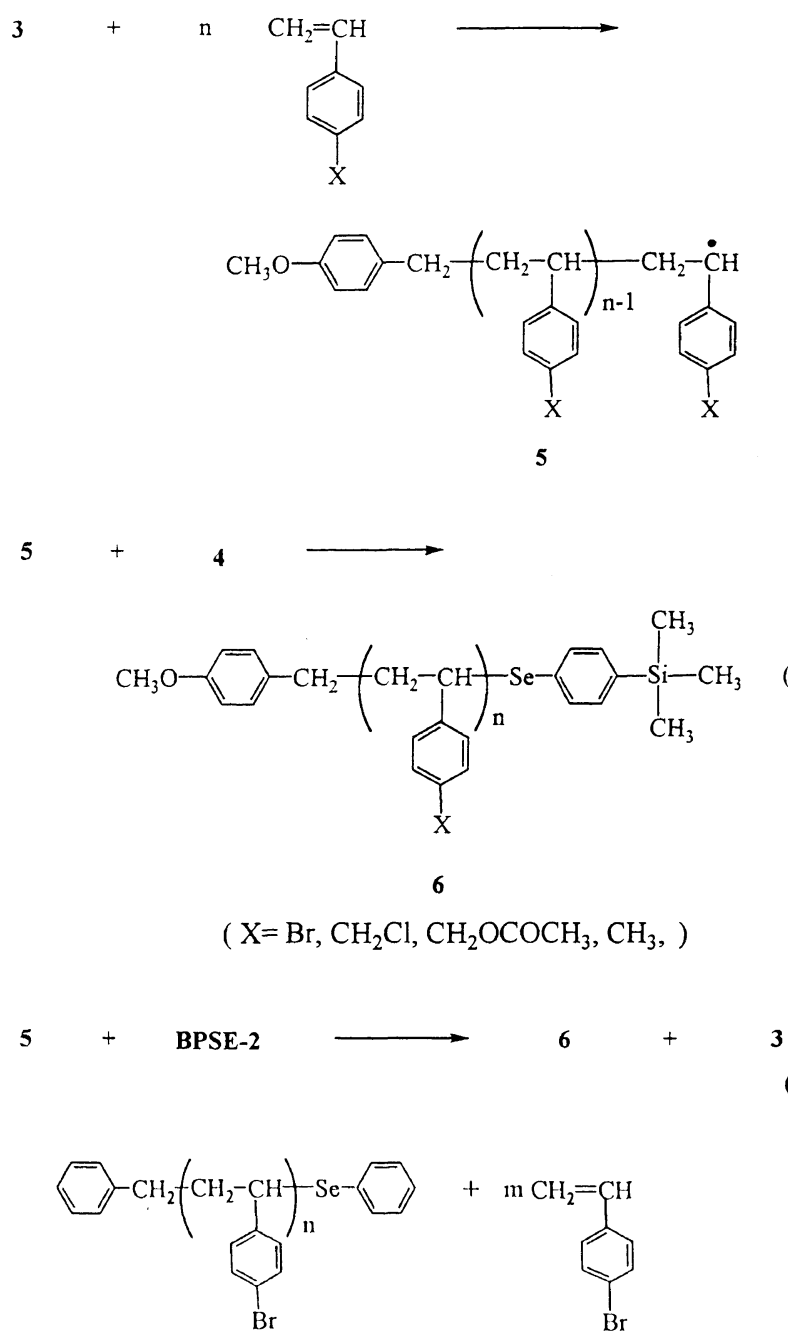

PBS-1

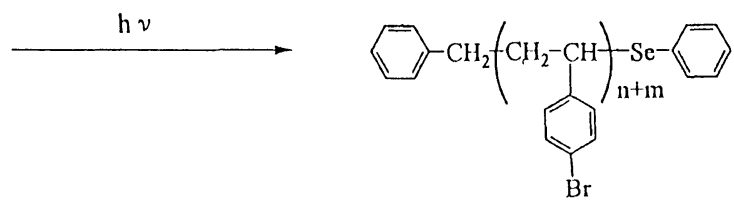

Polymerization was carried out by photoirradiation of solutions of $p$-substituted styrenes and BPSE-2 at room temperature (Table II). ${ }^{1} \mathrm{H}$ NMR spectra of the polymers (PMS-2, PBS-2, PVBAc-2, and PCMS-2) are shown in Figure 2. Signals due to trimethylsilyl and methoxy groups were observed at 0.25 and $3.72 \mathrm{ppm}$, respectively. The intensity ratio of the trimethylsilyl peak (b) to the methoxy peak (a) of all polymers was approximately 3 .

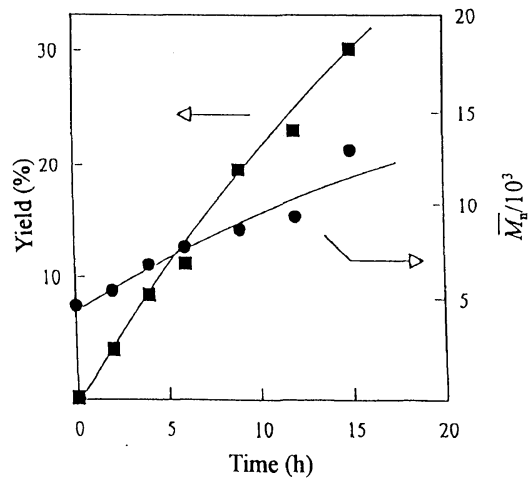

Figure 3. Time-yield $(\square)$ and time- $\bar{M}_{n}(\mathbf{O})$ relations for photopolymerization of BS with PBS-1 as polymeric photoiniferter. PBS- $1=0.1 \mathrm{~g}, \mathrm{BS}=1.5 \mathrm{~mL}$; benzene $=1 \mathrm{~mL} ;[\mathrm{BS}] /[\mathrm{PBS}-1]=1000$.

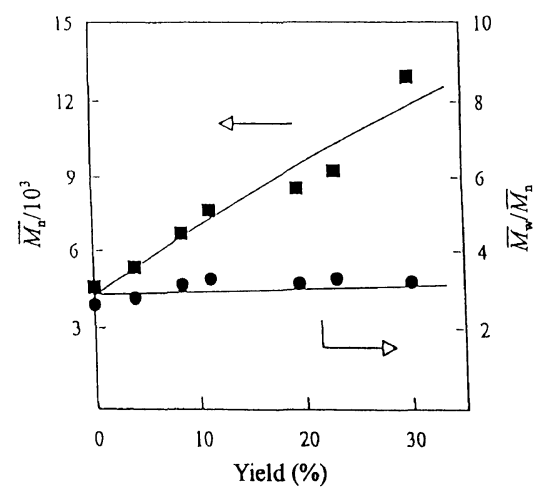

Figure 4. Yield $-\bar{M}_{n}(\square)$ and yield $-\bar{M}_{w} / \bar{M}_{n}$ (O) relations for photopolymerization of BS with PBS-1 as polymeric photoiniferter. PBS-1 = $0.1 \mathrm{~g} ; \mathrm{BS}=1.5 \mathrm{~mL}$; benzene $=1 \mathrm{~mL} ;[\mathrm{BS}] /[\mathrm{PBS}-1]=1000$.

For PMS-2, PVBAc-2, and PCMS-2, average functionality (DF) was calculated by comparing $\bar{M}_{n}$ from GPC with the peak intensity ratios of the trimethylsilyl signal (b) at $0.25 \mathrm{ppm}$ to methyl or methylene signals (f) of the side chain at $2.25 \mathrm{ppm}$ for PMS-2, 5.05 ppm for PVBAc-2, and $4.45 \mathrm{ppm}$ for PCMS-2, respectively. In the case of PBS-2, DF was obtained by comparing $\bar{M}_{n}$ from GPC with the peak intensity ratio of trimethylsilyl signal (b) to the sum of methylene (c) and methyne signals (d) at $1.25-1.85 \mathrm{ppm}$ in polystyrene backbone. DFs were calculated as $0.9-1.1$ (Table II). These values were reproducible to about $\pm 5 \%$ on repeated runs. This indicates that functionalized poly( $p$-substituted styrene)s prepared by this method contain benzyl and phenylseleno groups at $\alpha$ - and $\omega$-chain ends, respectively. The mechanisms are thus the same as in the previous report ${ }^{7}$ in which the monomer was inserted into the carbon-selenium bond (eq 3-5).

To confirm the preparation of end functional polymers, a chain extension experiment was made using PBS-1 $\left(M_{n}=4500, \bar{M}_{w} / \bar{M}_{n}=2.45, \mathrm{DF}=0.9\right) \quad($ eq 6$)$. When a solution of PBS-1 with BS was irradiated for the required reaction time, the yield and $\bar{M}_{n}$ of the polymer increased with reaction time as shown in Figure 3. $\bar{M}_{n}$ also increased with polymer yield as shown in Figure 4. Similar results were obtained from the chain extension experiment for other poly( $p$-substituted styrene)s. Poly( $p$-substituted styrene)s containing phenylseleno group as a reactive $\omega$-chain end can thus be prepared. 
Synthesis of AB-type Block Copolymers

Block copolymerizations of 1PS $\left(\bar{M}_{n}=4600, \bar{M}_{w} / \bar{M}_{n}=\right.$ $1.80, \mathrm{DF}=0.95)$ with $p$-substituted styrenes were investigated. The polymerization was carried out by ir-
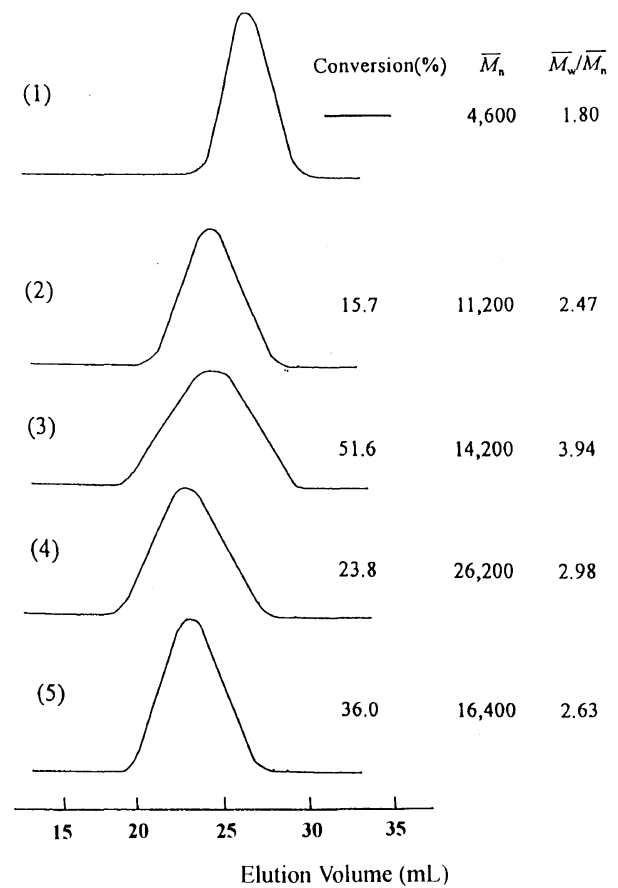

$26,200 \quad 2.98$

$6,400 \quad 2.63$

Figure 5. GPC profiles of (1) 1PS, (2) 1PS- $b$-PMS, (3) 1PS- $b$-1PBS, (4) 1PS- $b$-PVBAc, and (5) 1PS- $b$-PCMS. 1PS $=0.06 \mathrm{~g}$; $p$-substituted styrene $=3 \mathrm{~mL}$; benzene $=2 \mathrm{~mL} ;[p$-substituted styrene $] /[1 \mathrm{PS}]=1000$.
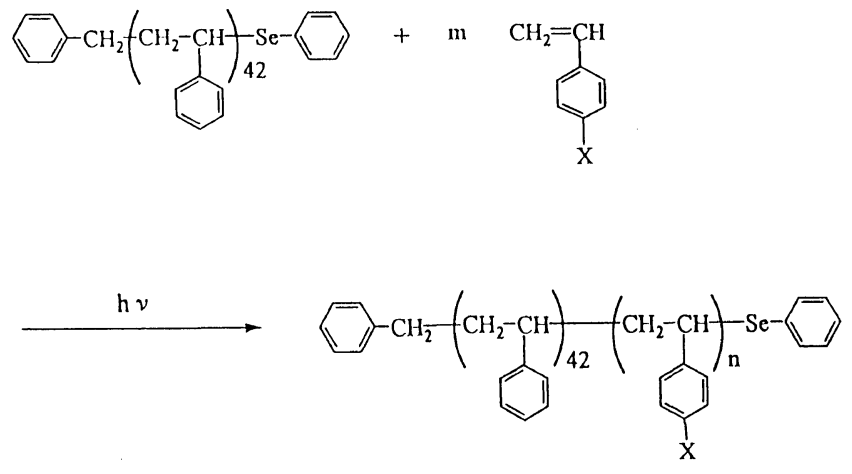

$\left(\mathrm{X}=\mathrm{Br}, \mathrm{CH}_{2} \mathrm{Cl}, \mathrm{CH}_{2} \mathrm{OCOCH}_{3}, \mathrm{CH}_{3}\right)$

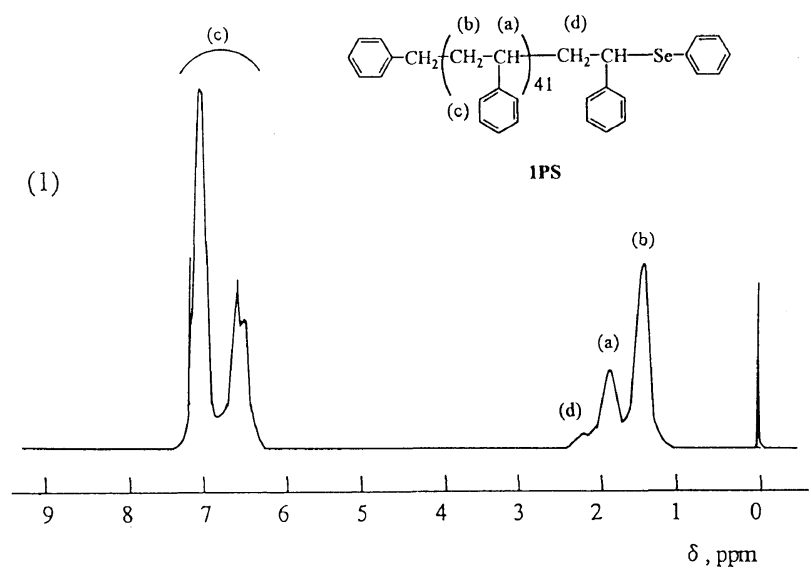

radiation of the solution of 1PS and $p$-substituted styrene in benzene at room temperature. Figure 5 shows the GPC curves of 1PS and resulting block copolymers. The curves of copolymers in case of all shifted to the higher molecular weight region in comparison with 1PS. These copolymers were unimodal in GPC curves, and the curve due to 1PS could hardly be observed on the

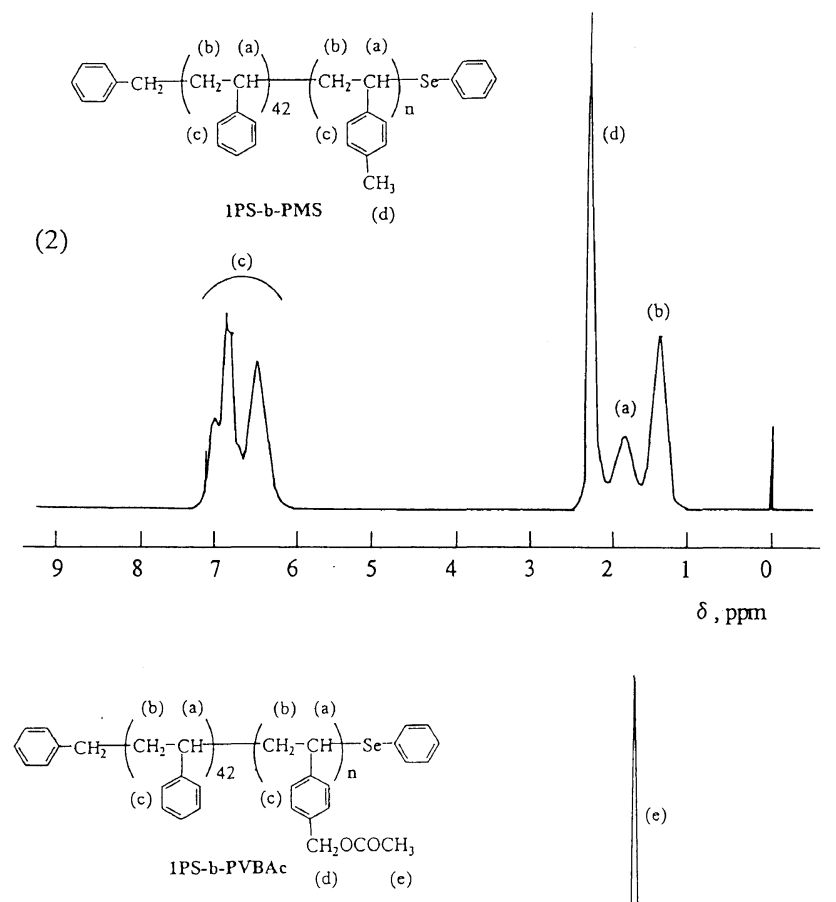

(3)
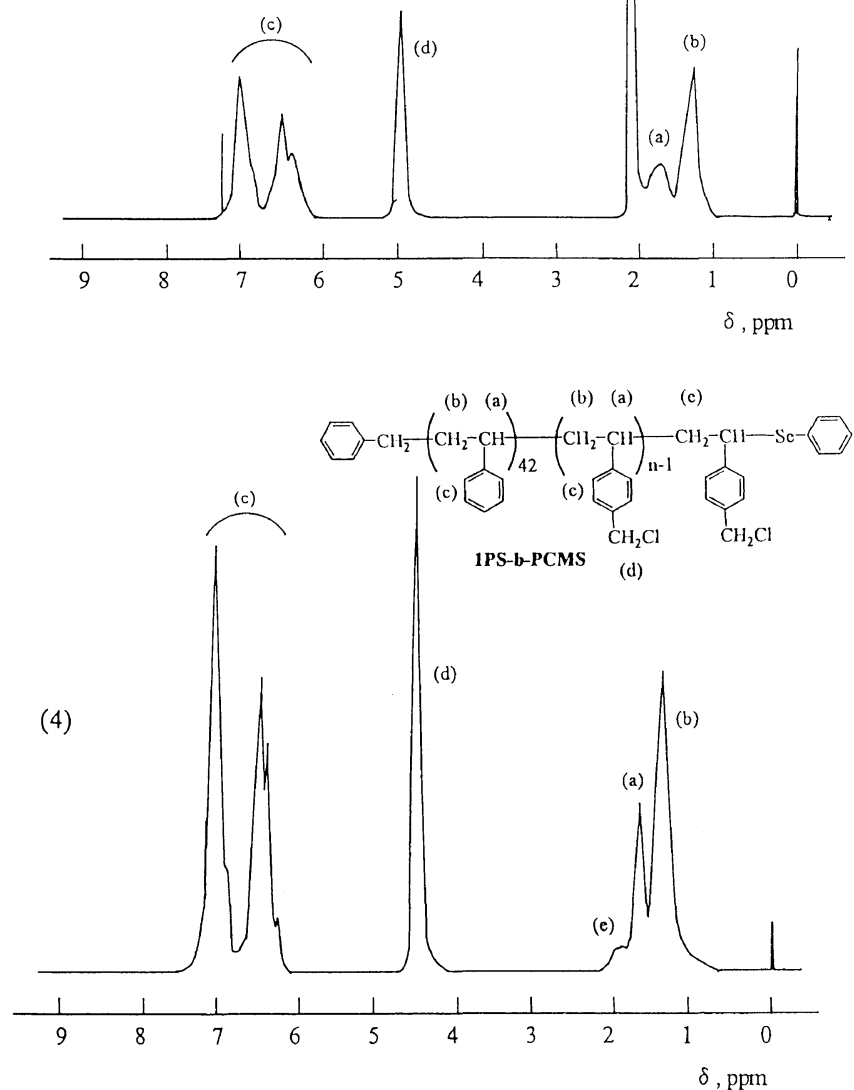

Figure 6. ${ }^{1} \mathrm{H}$ NMR spectra $\left(400 \mathrm{MHz}\right.$, in $\left.\mathrm{CDCl}_{3}\right)$ of (1) 1PS, (2) 1PS- $b$-PMS, (3) 1PS- $b$-PVBAc, and (4) 1PS- $b$-PCMS. 1PS $=0.06 \mathrm{~g}$; $p$-substituted styrene $=3 \mathrm{~mL} ;$ and benzene $=2 \mathrm{~mL} ; \quad[p$-substituted styrene $] /[1 \mathrm{PS}]=1000$. 
Table III. Preparation ${ }^{\mathrm{a}}$ of block copolymers from $1 \mathrm{PS}^{\mathrm{b}}$ with $p$-substituted styrene $\left(\mathrm{M}_{2}\right)$

\begin{tabular}{|c|c|c|c|c|c|c|c|}
\hline \multirow[b]{2}{*}{ Block copolymer ${ }^{\mathrm{c}}$} & \multirow{2}{*}{$\frac{\text { Time }}{\mathrm{h}}$} & \multirow{2}{*}{$\frac{\text { Yield }^{\mathrm{d}}}{\%}$} & \multirow[b]{2}{*}{$\bar{M}_{n} / 10^{3}$} & \multirow[b]{2}{*}{$\bar{M}_{w} / \bar{M}_{n}$} & \multicolumn{3}{|c|}{ Degree of polymerization of block copolymer } \\
\hline & & & & & by ${ }^{1} \mathrm{H} N M R^{e}$ & by GPC & by $\mathrm{Br}$ analysis \\
\hline $1 \mathrm{PS}-b$-PMS & 30 & 15.7 & 11.2 & 2.47 & $42: 63$ & $42: 63$ & \\
\hline 1PS- $b$-PBS & 30 & 51.6 & 14.2 & 3.94 & & $42: 40$ & $42: 51$ \\
\hline 1PS- $b$-PVBAc & 20 & 23.8 & 26.2 & 2.98 & $42: 126$ & $42: 123$ & \\
\hline 1PS- $b$-PCMS & 20 & 36.0 & 16.4 & 2.63 & $42: 82$ & $42: 77$ & \\
\hline
\end{tabular}

${ }^{\mathrm{a}}$ Irradiation of $100 \mathrm{~W}$ high pressure mercury lamp at room temperature. ${ }^{\mathrm{b}}$ 1PS (Polymeric photoiniferter), $0.092 \mathrm{~g}, \bar{M}_{n}=4600, \bar{M}_{w} / \bar{M}_{n}=1.80$ ${ }^{c}[p$-Substituted styrene $]=4.0 \mathrm{~mol} \mathrm{~L}-1$ in benzene solution. [ $p$-Substituted styrene $] /[1 \mathrm{PS}]=1000 .{ }^{\mathrm{d}} \mathrm{Yield} / \%=((\mathrm{Weight}$ of $\mathrm{precipitated}$ polymer - Weight of prepolymer)/Weight of charged monomer) $\times 100$, that is conversion of $p$-substituted styrene. ${ }^{\mathrm{e}} 400 \mathrm{MHz}$ in $\mathrm{CDCl}_{3}$.

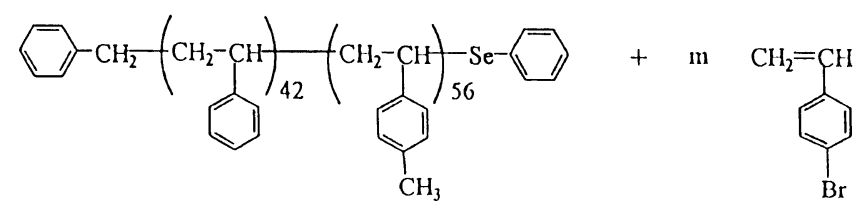

IPS- $b$-PMS

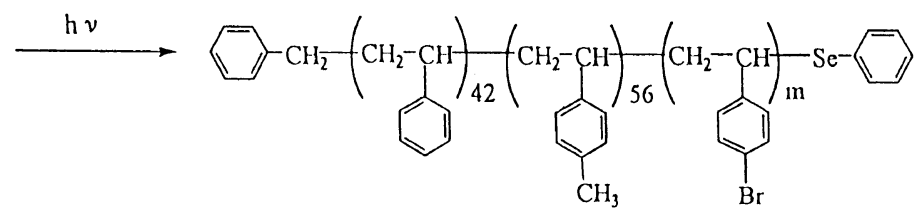

IPS- $b$-PMS- $b$-PBS

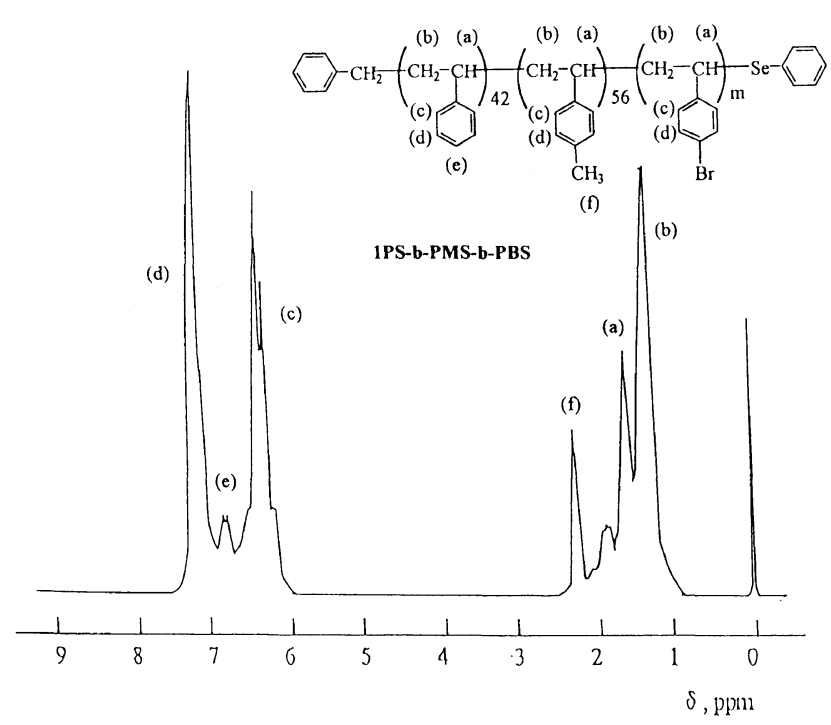

Figure 7. ${ }^{1} \mathrm{H}$ NMR spectrum $\left(400 \mathrm{MHz}^{2}\right.$ in $\left.\mathrm{CDCl}_{3}\right)$ of $1 \mathrm{PS}-b$-PMS$b$-PBS. 1PS- $b$-PMS $=0.075 \mathrm{~g}$; BS $=2 \mathrm{~mL}$; benzene $=3 \mathrm{~mL}$; [BS $] /[1 \mathrm{PS}-$ $b$-PMS] $=2000$.

GPC curve of the copolymers. No solvent combination that would separate block copolymers and homopolymers completely is presently available. But from above results, we thought that IPS would function as polymeric photoiniferter for the polymerization of $p$-substituted styrenes to afford the corresponding block copolymers as shown in eq 7 . However, $\bar{M}_{w} / \bar{M}_{n}$ of block copolymers was higher than that of IPS, and in case of the block copolymer of styrene with BS, that was widest. These block copolymers involving small amounts of homo- polymers of $p$-substituted styrenes are thus due to photopolymerization. The polymeric photoiniferter could not effectively act for polymerization of BS, and deactivation of larger portion in $p$-bromostyrene homopolymer would occur, compared to that of other $p$-substituted styrenes. The approximate composition of the block copolymer was determined from ${ }^{1} \mathrm{H}$ NMR (Figure 6), by comparing the relative ratio of methyl protons (d) in para site of $p$-substituted styrenes unit and phenyl protons (c) of the sum of styrene and $p$-substituted styrenes units. Because the peak from BS unit did not appear, elementary analysis of bromine method was used in case of the block copolymer of styrene and BS. The results are shown in Table III. The values quite agree with those obtained by GPC measurement. But small difference in case of 1 PS- $b$-PBS was observed. It is thought from inherent in the elementary analysis of bromine method. From the above GPC and ${ }^{1} \mathrm{H}$ NMR results, the AB-type block copolymer can be effectively prepared using the polystyrene containing seleno group at $\omega$-chain end as polymeric photoiniferter in the presence of $p$-substituted styrenes.

\section{Synthesis of ABC-Type Block Copolymer}

The photopolymerization of BS with the AB-type block copolymer of styrene and MS (1PS- $b$-PMS, $\bar{M}_{n}=$ $11200, \bar{M}_{w} / \bar{M}_{n}=2.50$, numbers of St and MS units are 42 and 56, respectively) as polymeric photoiniferter in benzene was carried out as shown in eq 8 . A triblock copolymer (1PS- $b$-PMS- $b$-PBS) was obtained with $\bar{M}_{n}=$ $22000, \bar{M}_{w} / \bar{M}_{n}=2.70$ by GPC. This shows the number of PBS unit is 60. Figure 7 shows the ${ }^{1} \mathrm{H}$ NMR spectrum 
of 1 PS- $b$-PMS- $b$-PBS. By comparing the intensity ratios of methyl protons (f) at $2.3 \mathrm{ppm}$ of the $p$-methylstyrene and phenyl protons at $6.0-7.3 \mathrm{ppm}$ of the 1PS- $b$-PMS$b$-PBS, the numbers of St, MS, and BS units in triblock copolymer were obtained 42,56 , and 65 , respectively. These values were approximately obtained by GPC (42/56/60). The AB-type block copolymer thus functions as polymeric photoiniferter, and it should be possible to obtain the ABC-block copolymer.

\section{CONCLUSIONS}

BPSE- 1 acted as a photoiniferter for the polymerization of BS, CMS, MS, and VBAc. Based on the end structures of poly ( $p$-substituted styrene)s obtained with BPSE-2, the polymer contains benzyl and arylseleno groups at $\alpha$ - and $\omega$-chain ends, respectively. Photoirradiation of the polystyrene containing phenylseleno group at the $\omega$-chain end as polymeric photoiniferter in the presence of $p$-substituted styrene afforded AB-type block copolymers of styrene and $p$-substituted styrenes.

Acknowledgments. This work was supported by Grant-in-Aid for Scientific Research (No. 10650865) from the Ministry of Education, Science, Sports and Culture of Japan.

\section{REFERENCES}

1. S. Patai and Z. Rappoport, "The Chemistry of Organic Selenium and Tellurium Compounds," Wiley, New York, N.Y., 1986.

2. K. C. Nicolaou, Tetrahedron, 37, 4097 (1981).

3. D. Liotta, Acc. Chem. Res., 17, 28 (1984).

4. T. Ando, T. S. Kwon, A. Kitagawa, T. Tanemura, S. Kondo, H. Kunisada, and Y. Yuki, Macromol. Chem. Phys., 197, 2803(1996).

5. T. S. Kwon, S. Kondo, H. Kunisada, and Y. Yuki, Eur. Polym. $J .$, in print.

6. T. S. Kwon, S. Kumazawa, T. Yokoi, S. Kondo, H. Kunisada, and Y. Yuki, J. Macromol. Sci., Pure Appl. Chem., A34, 1553 (1997).

7. T. S. Kwon, S. Kondo, H. Kunisada, and Y. Yuki, Polym. J., 30, 559 (1998).

8. T. S. Kwon, S. Kondo, H. Kunisada, and Y. Yuki, unpublished results.

9. J. Farral and J. M. Frechet, J. Org. Chem., 41, 3877 (1976).

10. Y. Guibert and J. Brossas, Polym. Bull., 1, 293 (1979).

11. A. Lewis and J. Azoro, J. Org. Chem., 46, 1764 (1981).

12. H. R. Allcock, T. J. Fuller, and T. L. Evans, Macromolecules, 13, 1325 (1980).

13. M. Gauthier, M. Moller, and W. Burchard, Macromol. Symp., 77, 43 (1994).

14. J. G. Abrams and E. C. Chapin, J. Org. Chem., 26, 2671 (1961).

15. C. H. Bamford and H. Lindsay, Polymer, 14, 330 (1973).

16. D. T. Mowry, M. Renoll, and W. F. Huber, J. Am. Chem. Soc., 68, 1105 (1946).

17. W. S. Emerson, J. W. Heyd, V. E. Lucas, W. I. Lyness, G. R Owens, and W. Shortridge, J. Am. Chem. Soc., 69, 1905 (1947). 